\title{
EV 装置中电子等离子体对低频信号的传递
}

\author{
郑 少白 \\ (中国科学院物理研究所, 北京 100080 )
}

\author{
J. H. Malmberg, C. F. Driscoll
}

(美国加州大学弪地分校物理系,洛杉䂗)

摘要

本文对 $\mathrm{EV}$ 装置中非中性电子等离子体对低濒信号的传递做了实验、理论及数 直计算的研究, 三者结果非常一致。这说明 EV 装置中电子等离子体对低频信号的 传递是属于总体运动的结果。同时本文结果可作为一种测量类似 EV 装置中非中性 电子等离子体总能量的简单方法.

\section{关键词：电子等密子体，低频调制}

\section{一、引 言}

非中性等离子体是等离子体学科中的重要一支. 由于龙分单纯, 长时间约束及物理机制 相对简单的特点, 它比较容易用来验证一些等离子体的基本理论. 经过 Malmberg 和 deGerassie $^{[1]}$ 的开创性工作之后, 非中性电子等离子体的实验和理论工作得到迅速的发展 ${ }^{[2-5]}$. 约 策时间达几小时的非中性电子等离子体装置 $\mathrm{EV}^{[6]}$ 就是最新最有代表性的实验装咭.

和本文有关的 $\mathrm{EV}$ 装置的实检安排如图 1. 主要部分是由 3 节同轴圆筒组成. 内径 $R=$ $3.8 \mathrm{lcm}$. 两边两节 $A_{1}, A_{2}$ 分别叫注人筒和泄放筒, 都是 $7.62 \mathrm{~cm}$ 长, 平时接堡电位 $V_{1}$ 和 $V_{2}$. 中 间节 $A_{0}$ 为工作筒，长 $34.3 \mathrm{~cm}$, 在实验中接地. 3 节圆筒处于非常均匀的轴泃磁场 $B$ 中. 系 统的本底真空为 $10^{-8} \mathrm{P}_{\mathrm{d}}$ 。实验的工作周期由注人、保持、泄放/淤星 3 个环节组成。开始时 $A_{1}$ 输人一个正的注入䟿冲电压。这样在 $A_{1}$ 左边为负电位 $V_{b}$ 的灯丝发射的电于无阻拦地 注入工作筒中。调节灯丝的电位叮改变筒中电子等离子体的密淁和能量. 当注人脉冲过后, 工作筒内电子的横向运动被磁场约串. 它的纵向运动被 $A_{1}, A_{2}$ 筒的负电位封锁. 因此电子. 离子体可长时间保持在工作筒内. 最后 $A_{2}$ 加一正的泄放脉冲电压,筒内的电子沿磁力线问 右泄出，这样筒内的总电子数 $N_{0}$ 可用主 Faraday 筒接收测得. 同时它有一个可改变径向位 筧的小孔. 沿磁力线通过小孔的电子可用副 Faraday 筒记录,从而可计算得沿磁力线积分的 电子线密度分布 $N(r)$. 同时用一磁分析器测得该束电子垂直于磁场方问的动能. 由于装留 中同一磁力线上的电子之间碰墥弛豫时间 ( $\simeq 1 \mathrm{~ms}$ ) 远短于电子在筒内的约束时间. 电子平 


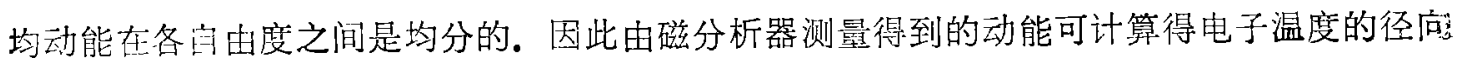
分布 $T(r)$.

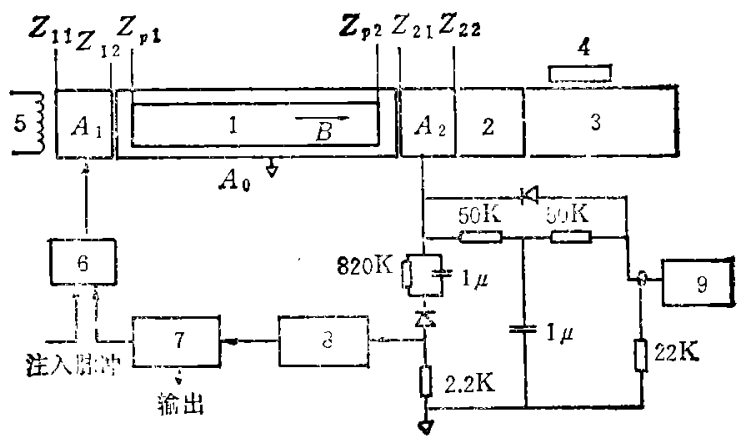

图 1 实验安排

( $A_{1}$ 注入筒， $A_{2}$ 泄放筒， $A_{0}$ 工作筒；1-电子等离子体，2-主 Faraday 筬，3-一副 $\mathrm{F}$ -

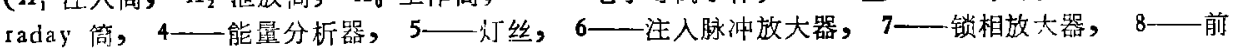

犆放大器，9-—泄放脉冲发生器）

在电子等离子体保持在工作筒 $A_{0}$ 内时, 注人筒 $A_{1}$ 叠加一个低频调制电压信号, 同时保持 $A_{2}$ 的电位不变. 那么 $A_{2}$ 要出现交变的感应电荷 (下面简称为调制电荷). 本文即是研究这调 制电荷和调制电压之间的关系。下面的实验和理论表明调制电荷是正比于电子等离子体的总 能量. 因此可用该方法简单地,不破坏等离子体的状态下测量等离子体的总能量.

\section{二、实验测量方法和结果}

恕图 1 , 调制信号是由恍相放大器的标准信号输出经注人脉冲放大器后加到注入筒 $A_{1} . A_{\star}$ 的调制电荷原则上可用测量接地电阻上的交流压降而得到. 为了尽可能减少测量误差, 该交 流电医要非常小 (约 $\left.10^{-10} \mathrm{~V}\right)$. 所以我们用良好的前量放大器和锁相放大器来消除测量中的噪 音. 另一方面在装置的注人和泄放工作期间有一个很强的干扰进入锁相放大器, 为此我们设 计了如图 1 所示的专门测量线路。这样可以保证锁相放大器在注入和浛放期间不致于被惿。

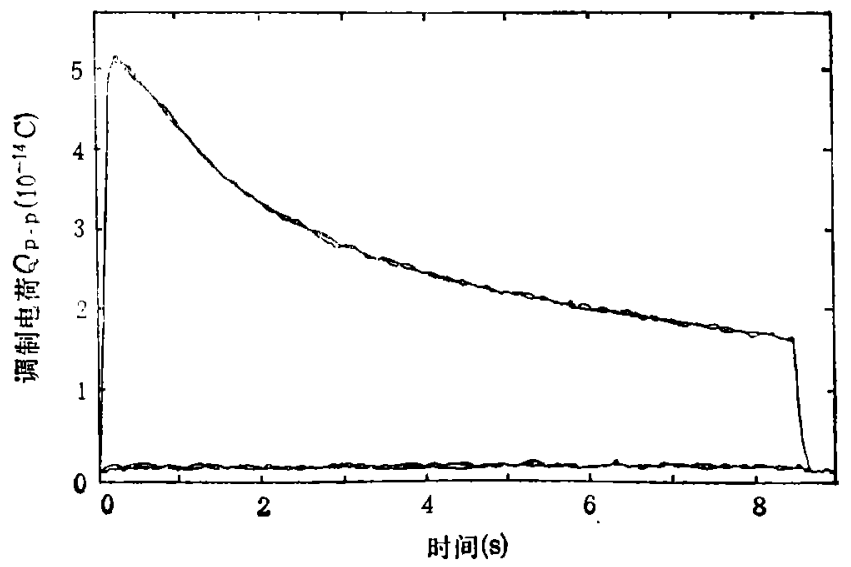

图 2 典型的调制电荷信号 


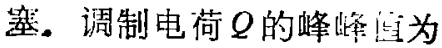

$$
Q_{\mathrm{p}-\mathrm{p}}=2 \sqrt{2} \frac{a}{R \underline{Q} \omega} V,
$$

这里 $\alpha$ 为前置放大器的放大倍数, $R Q$ 为负载电阻 $(2.2 \mathrm{k} Q)$. $\omega$ 为调制信号元频率, $V$ 为锁相 故大器显示的电压有效值. 图 2 是贵型的测量结果曲线. 实验条件是 $V_{1}=V_{2}=-60 \mathrm{~V}$. 磁 场 $B=94 \times 10^{-4} \mathrm{~T}$. 灯丝偏压 $V_{b}=-30 \mathrm{~V}$. 调制信号频率为 $40 \mathrm{kC}$, 电压有效值为 $2 \mathrm{~V}$, 在 0 - $8.5 \mathrm{~s}$ 期间加人. 曲线是由多次实验重复在记录仪上画得, 为了比较, 图 2 同时给出无等离 子体时的调制电荷曲线, 可以看出无等离子体时信号为零. 在实验中为了便于在秒量级时间 内观察到不问等离子体参数对实验的影响, 因此人为地加人一些杂散磁场使等离子体寿命变 短. 表 1 给出了不同时刻的总电子数,平均温度及调制电荷的峰峰值.

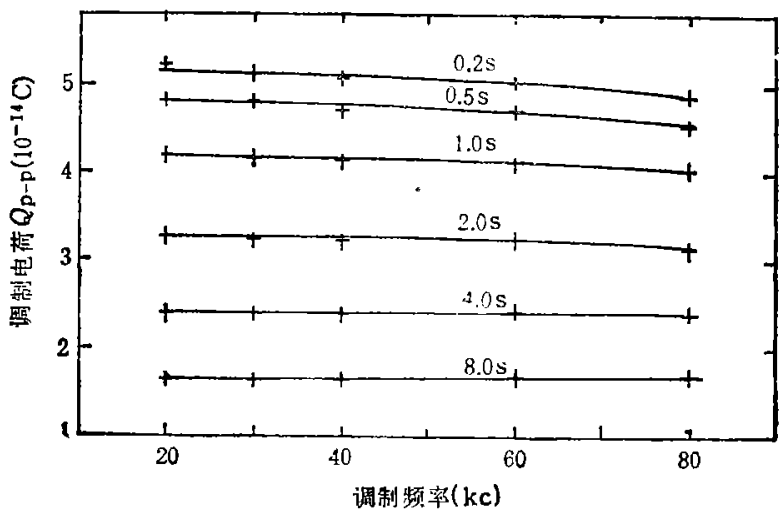

图 3 调制电荷和调制频率的关系

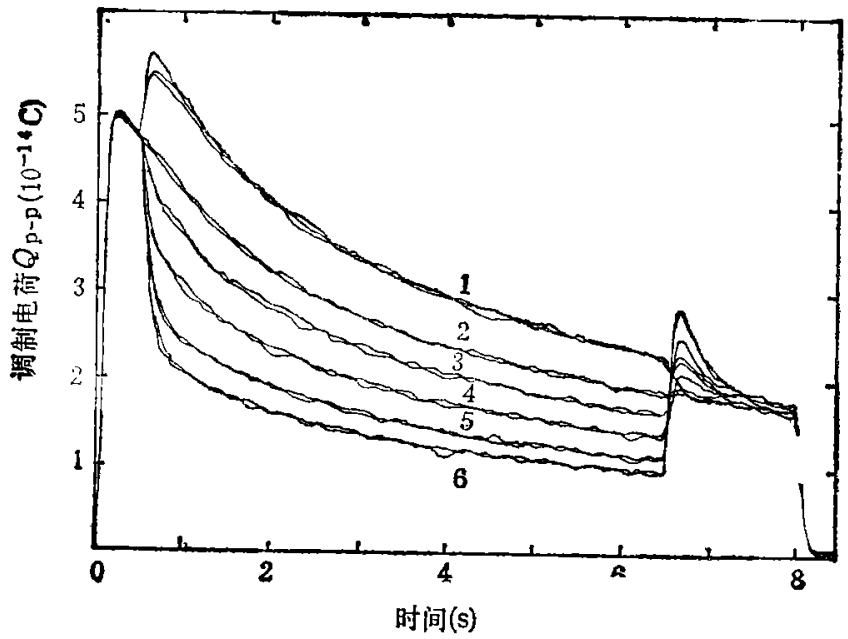

图 4 不同 $V$ 、下的调制电荷信号

$\left(1-V_{1}=-50.4,2--59.8,3--69.1,4--82.8,5\right.$ $-105.8,6--128.0)$

图 3 给出了在相同电子等离子体条件下不同调制频滈的调制电菏. 从图 3 中可以看出调 证电何和频率基本上没有关系。同时也说明等离子体对信号传递的响位频率要高于 $80 \mathrm{kC}$. 
表 1 典型条件下电子等离子体数据及调制电荷

\begin{tabular}{|c|c|c|c|c|c|c|c|}
\hline & & $\begin{array}{l}60 \mathrm{~V} \\
60 \mathrm{~V}\end{array}$ & $\begin{array}{l}V_{b}=-30 \mathrm{~V} \\
B=94 \times 10^{-} \mathrm{T}\end{array}$ & $\begin{array}{l}\text { 调制频 } \\
\text { 调制电! }\end{array}$ & $\begin{array}{l}0 \mathrm{kC} \\
x^{2}\end{array}$ & & \\
\hline \multicolumn{2}{|c|}{ 采样时间 $t(s)$} & 0.2 & 0.5 & 1.0 & 2.0 & 4.0 & 8.0 \\
\hline \multicolumn{2}{|c|}{ 总电子数 $N_{0}\left(10^{9}\right)$} & 3.15 & 3.06 & 2.67 & 2.05 & 1.50 & 1.07 \\
\hline \multicolumn{2}{|c|}{ 平均温度 $(\mathrm{eV})$} & 2.78 & 3.66 & 4.22 & 4.87 & 5.51 & 5.58 \\
\hline \multicolumn{2}{|c|}{ 中心电位 $\phi(0,0)(\mathrm{eV})$} & -23.7 & -20.5 & -17.2 & -13.7 & -10.7 & -8.32 \\
\hline \multicolumn{2}{|c|}{ 平均电位 $\phi_{0,0}(\mathrm{eV})$} & -16.4 & -13.5 & -11.1 & -9.07 & -7.33 & -5.93 \\
\hline 调制电荷 & 实验值 & 5.11 & 4.81 & 4.19 & 3.27 & 2.40 & 1.69 \\
\hline 峰峰值 & 近似理论 & 4.70 & 4.52 & 3.83 & 2.93 & 2.68 & 1.82 \\
\hline$Q_{p-p}\left(10^{-14} \mathrm{C}\right)$ & 数值计算 & 4.81 & 4.38 & 3.72 & 2.95 & 2.22 & 1.60 \\
\hline
\end{tabular}

为了在保持其他条件不变下观察 $A_{1}$ 上封锁电压 $V_{1}$ 对调制电荷的影响, 我们在等离子体 “保持到 $0.5 \mathrm{~s}$ 时突然改变 $V_{1}$ 值．对于不同的 $V_{1}$ 改变值的测量结果如图 4. 计算结果表明调制 电荷是反比于封锁电压 $V_{1}$.

实验还发现调制电荷和其他参数的关系很好符合下一节的理论公式,这里不再一一列举.

\section{三、近似理论}

如图 1, 轴对称的圆筒内半径为 $R, A_{1}, A_{2}$ 两端轴向坐标分别为 $Z_{11}, Z_{12}, Z_{21}, Z_{22}$. 筒 $A_{0}$ 和 $A_{1} A_{2}$ 之间的间隙可忽视. 由于 $A_{0}$ 接地, 因此筒内的电位 $\phi(r, Z)$ 是由 $A_{1}, A_{2}$ 及等离子体 产生的电位的叠加. 它们分别记 $\phi_{1}(r, Z), \phi_{2}(r, Z)$ 及 $\phi_{0}(r, Z)$. 有

$$
\phi(r, Z)=\phi_{1}(r, Z)+\phi_{2}(r, Z)+\phi_{0}(r, Z),
$$

其中 $\phi_{1}, \phi_{2}$ 分别正比于 $V_{1}$ 和 $V_{2}$. 设筒内电子密度分布为 $n(r, Z)$, 由 Stokes 定律可得到 电子等离子体在 $A_{2}$ 上感应的像电荷为,

$$
q=\frac{2 \pi e}{V_{2}} \int_{0}^{R} \int_{Z_{12}}^{z_{21}} n(r, Z) \phi_{2}(r, Z) r d r d Z \text {. }
$$

由于筒 $A_{0}$ 细而长, $\left(Z_{21}-Z_{12}\right) \gg R$. 这样在 $A_{0}$ 内绝大部分区域有 $\phi_{1}=\phi_{2}=0$, 只有 在端部附近不为零并有很大的梯度. 因此在计算中近似假定电子等离子体是一个在 $Z$ 方向均 匀分布的圆柱, 两端面位置分别为 $Z_{p_{1}}, Z_{p_{2}}$. 圆柱内电子密度分布为 $n(r)$. 将电位 $\phi_{1}, \phi_{2}$ 在 $z$ 方向分布用 Bessel 函数展开. 在只取零阶项近似下,在近工作筒两端部筒内的电位分布有

$$
\begin{aligned}
& \text { 左端: } \phi_{2}=0, \phi_{1}(r, Z)=V_{1} e^{-\frac{z-Z_{p_{12}}}{\rho}} Z \subsetneq Z_{12}, \\
& \text { 右端: } \phi_{1}=0, \phi_{2}(r, Z)=V_{2} e^{-\frac{z_{21}-Z}{\rho}} Z \approx Z_{21},
\end{aligned}
$$

这里 $\rho$ 是衰减长度. 利用筒壁电位为零的边界条件可得到 $\rho=R / 2.4$. 如果知道等离子体内 电子在 $\mathrm{Z}=0$ (工作筒中心)处的电位和平行于轴向的动能 $W_{\|}(r)$. 由于电子在工作筒内来 回反弹时间比碰撞弛豫时间小得多, $Z_{p_{1}}, Z_{p_{2}}$ 位置满足:

$$
\begin{aligned}
W_{n}(r)-e \phi_{0}(r, 0) & =-e\left[\phi_{1}\left(r, Z_{p_{1}}\right)+\phi_{0}\left(r, Z_{p_{1}}\right)\right] \\
& =-e\left[\phi_{2}\left(r, Z_{p_{1}}\right)+\phi_{0}\left(r, Z_{p_{2}}\right)\right] .
\end{aligned}
$$

由于等离子体为电子均匀分布的细长圆柱的假设, $\phi_{0}$ 满定 $\phi_{0}\left(r, Z_{p_{1}}\right)-\phi_{0}\left(r, Z_{p_{2}}\right) \cong$ 
$\frac{1}{2} \phi_{0}(r, 0)$, 代人(5)式有

$$
\phi_{1}\left(r, Z_{p_{1}}\right)=\phi_{2}\left(r, Z_{p_{2}}\right)=\frac{1}{2} \phi_{0}(r, 0)-\frac{1}{e} W_{\prime \prime}(r),
$$

在筒 $A_{2}$ 上的感应电荷为

$$
q=\frac{2 \pi e}{V_{2}} \int_{0}^{R} n(r) r d r \int_{z_{p_{1}}}^{Z_{p_{2}}} \phi_{2}(r, Z) d Z
$$

等离子体的长度

$$
L=Z_{\rho_{3}}-Z_{p_{1} \cdot}
$$

当筒 $A_{1}$ 的电位 $V_{1}$ 叠加调制电压 $\delta V_{1}\left(\left|\delta V_{1}\right| \ll\left|V_{1}\right|\right)$ 时, $Z_{p_{1}}, Z_{p_{2}}$ 要发生改变 $\delta Z_{p_{1}}$, $\delta Z_{p_{2}}$,

$$
\delta L=\delta Z_{p_{2}}-\delta Z_{p_{1}} .
$$

由于调制信号的周期比等离子体寿命短得多. 在一个调㭊周期内, 沿磁力线积分的电子 线密度分布 $N(r)$ 不变. 再加上等学子体细长圆杜的假设. $\phi_{0}(r, 0)$ 是正比于电子密度分 布:

$$
\frac{\delta \phi_{0}(r, 0)}{\phi_{0}(r, 0)}=\frac{\delta n(r)}{n(r)}=-\frac{\delta L}{L} .
$$

在一个调制周期之内, 电子平行于磁场和重:直于磁场的能量来不及交换。电子平行于磁 场动能的改变属于一维绝热压缩的过程.

$$
\frac{\delta W_{\| 1}(r)}{W_{\|}(r)}=-2 \frac{\delta L}{L} .
$$

将(4),(6)式微分, 代入(9)式,并利用(10),(11)式的结果可得到

$$
\delta L=\frac{\rho}{1+\frac{2 \rho}{L} \frac{e \phi_{0}(r, 0)-4 W_{\| \prime}(r)}{e \phi_{0}(r, 0)-2 W_{\| \prime}(r)}} \frac{\delta V_{1}}{V_{1}} .
$$

将(3)式微分, 可得 $A_{2}$ 上像电荷的改变。

$$
Q=\delta q=\frac{2 \pi e \delta V_{1}}{V_{1} V_{2}^{-}} \int_{0}^{R} \frac{\rho^{2}}{L^{2}} \frac{\phi_{0}(r, 0)-3 W_{\| \prime}(r) / e}{1+\frac{2 \rho}{L} \frac{e \phi_{0}(r, 0)-4 W_{\| l}(r)}{e \phi_{0}(r, 0)-2 W_{\| \prime}(r)}} N(r) r d r .
$$

考虑到 $\rho / L \ll 1$, 调制电荷和调制电压之间有非常简单的近似关系:

$$
\begin{aligned}
Q & =\frac{2 \pi}{V_{1} V_{2}} \delta V_{1} \int_{0}^{R} \frac{\rho^{2}}{L^{2}}\left[e \phi_{0}(r, 0)-3 W_{11}(r)\right] N(r) r d r \\
& =-\frac{1}{V_{1} V_{2}} N_{0}\left(E-e \phi_{00}\right) \delta V_{1},
\end{aligned}
$$

这里 $N_{0}$ 为总电子数,

$$
E-e \phi_{\infty 0}=\frac{2 \pi}{N_{0}} \int_{0}^{R}\left[3 W_{\|}(r)-e \phi_{0}(r, 0)\right] N(r) r d r .
$$

由于在 $E V$ 装置内, $W_{u}(r)=\frac{1}{2} W_{\perp}(r)$. 因此 $E-e \phi_{00}$ 为电子的平均能量. 这样摆制产 生的像电荷反比于 $V_{1}, V_{2}$, 正比于等离子体的总能量. 由于 $V_{1}, V_{2}$ 可由实验测量得到. 因此 
测量调制电荷即可计算得电子等离子体的总能量,而不需要破坏等离子体的状态.

从第二节可知, 在实验中我们可以测得等离子体的线密度分布 $N(r)$ 和温度的径向分布 $T(r)$. 利用下一节计算机解 Poisson 方程的结果可得到 $\phi_{0}(r, Z)$. 这样可得到 $\phi_{00}, E, N_{0}$. 代人(14)式可得到 $Q$. 表 1 给出在该条件下的 $N_{0}, E, \phi(0,0), \phi_{00}$ 及 $Q$ 值. 从表 1 可以看出 实验和近似理论结果符合得相当满意。图 5 给出以实验值为横坐标, 近似理论值为纵坐标, 在 不同的实验条件下的结果。看来两者相差约在 $10 \%$ 之内. 同时发现当 $V_{1}, V_{2}$ 的数值远大于. 灯丝偏压 $V_{b}$ 时,两者符合较好. 这可能是由于(4)式的近似条件满优较好的结果.

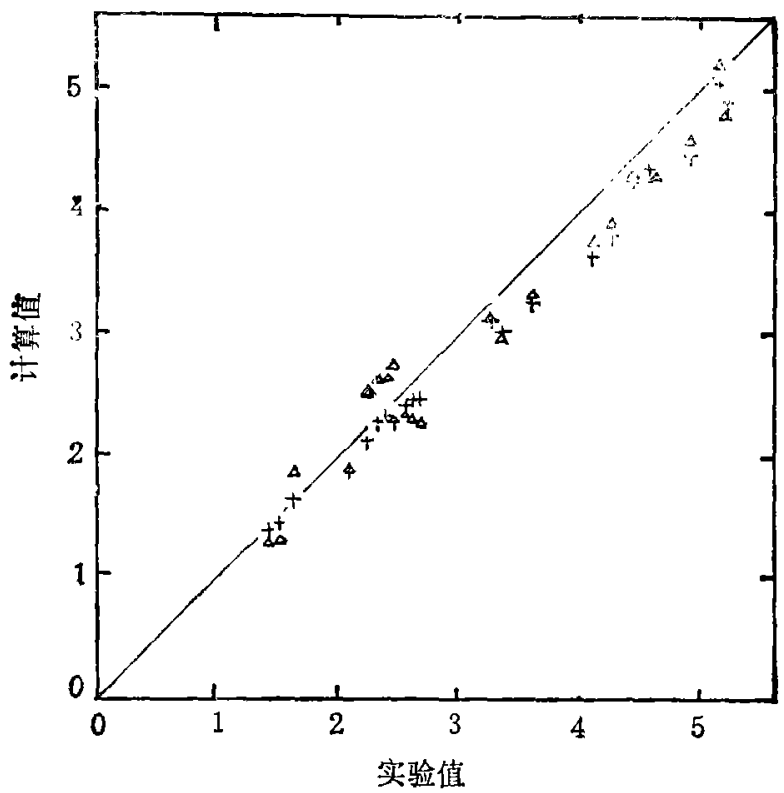

图 5 感应电荷的近似理论, 数值计算和实验结果的比较

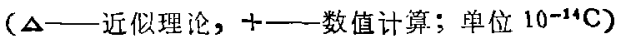

\section{四、严格的数值计算}

在上一节的近似理论计算中我们使用了一些如等离子体为纵向均匀的直圆柱, 在 $A_{0}$ 端部 $\phi_{1}, \phi_{2}$ 为(4)式等的近似假设. 为了验证这些假设的近似性, 我们对实验数据作了计算机的严 格的数值计算. 方法如下:

如果已知道筒内的电子等离子体密度分布 $n(r, Z)$, 那么就可解 Poisson 方程及边条作: 得到空间电位分布 $\phi(r, Z)$.

$$
\begin{aligned}
& \nabla^{2} \phi(r, Z)=-4 \pi e n(r, Z), \\
& \phi(R, Z)= \begin{cases}V_{1} & Z_{11}<Z<Z_{12}, \\
0 & Z_{12}<Z<Z_{21}, \\
V_{2} & T_{21}<Z<Z_{22} .\end{cases}
\end{aligned}
$$

因此 $A_{2}$ 的总感应电荷, 即 $A_{2}$ 内表面的表面电荷可由 $A_{2}$ 内表面的电位梯度计算得到. 利 用实验中测得的电子温度 $T(r)$ 及线密度分布 $N(r) . n(r, Z)$ 满足的方程为: 


$$
\begin{aligned}
& \int_{Z_{11}}^{Z_{22}} n(r, Z) d Z=N(r), \\
& n(r, Z)=n_{0}(r) e^{\frac{e \phi(r, Z)}{T(r)}},
\end{aligned}
$$

这里 $n_{0}(r)$ 为待定系数。

将(16)式进行分离变数。在 $z$ 方向用快速 Fourier 变换, 在 $r$ 方向用差分法处理. 并用 多次迭代来求得满足方程 (18),(19)的解

由于调制产生 $A_{2}$ 上的调制电荷比起总的感应电荷要少得多. 因此不能简单地用改变 (17)式边条件, 求得新的电位分布,然后用两个电位之差来计算调制之后产生的调制电荷. 为 此我们采用直接计算密度和电位改变量的方法。

当 $A_{1}$ 加调制电压 $\delta V_{1}$, 那么空间的电位及密度改变 $\delta \phi(r, Z)$ 和 $\delta n(r, Z)$ 它们满足的 Poisson 方程及边界条件为

$$
\begin{aligned}
& \nabla^{2} \delta \phi(r, Z)=-4 \pi e \sin (r, Z), \\
& \delta \phi(R, Z)=\left\{\begin{array}{cc}
\delta V_{1} & Z_{11}<Z<Z_{12}, \\
0 & Z_{12}<Z<Z_{22} .
\end{array}\right.
\end{aligned}
$$

$V_{1}$ 电位改变之后的电子密度分布满足

$$
\begin{aligned}
n^{\prime}(r, Z) & =n(r, Z)+\delta n(r, Z) \\
& =\left[n_{0}(r)+\delta n_{0}(r)\right] e^{\frac{\sigma[\phi(r, Z)+\delta \phi(r, Z)]}{T(r)+\delta T} n^{(r)}},
\end{aligned}
$$

有

$$
\delta n(r, Z)=n(r, Z)\left[\left(1+\frac{\delta n_{0}(r)}{n_{0}(r)}\right) e^{\left\{\frac{\varepsilon[\phi(r, Z)+\delta \phi(r, Z)]}{T(r)+\hat{o} T} \eta^{(r)}-\frac{\varepsilon \phi(r, Z)}{T(r)}\right\}}-1\right],
$$

这里 $\delta T_{\|}(r)$ 是由于 $V_{1}$ 改变 $\delta V_{2}$ 引起电子在平行于磁场方向的温度改变. 和 $(11)$ 式有相同 琹由,

$$
\frac{\delta T_{l l}(r)}{T(r)}=\frac{2 \delta n(r, 0)}{n(r, 0)} .
$$

由于在一调制周期内, 电子线密度守恒:

$$
\int_{Z_{11}}^{z_{22}} \delta n(r, Z) d Z=0
$$

这样利用非调制的 $n(r, Z), \phi(r, Z)$ 值直接计算 $\delta \phi(r, Z)$ 有较高的精度. 计算方法 类似方程 (16)-(19)的求解. 对于表 1 中县型参数的计算结果列表于 1 中最后一行. 同样, 在各种不同条件下的结果在图 5 中给出. 我们可以看到严格的数值计算结果和近似理论结果 符合相当地好。

\section{五、结 论}

1. 实验结果, 近似理论及数值计算的结果之间的误差一般不大于 $10 \%$ 。这在等离子体测 量中是一个符合得非常婑的结果。说明电子等离子体对低频信号的传递是简单的整体运动的 过程.

2. 近似理论 (14) 式中主要的近似是等离子体两端形状及密度分布的假定及 $\rho / L \ll 1$ 的 近似. 我们作了进一步修正的计算之后, 发现修正后的理论值更接近于数值计算的结果, 但修 
正量不大于 3 - $5 \%$. 因此进一步的修正是一个小量.

3. 近似理论 (14)式中除电子等离子体总能量 $N_{0}\left(E-e \phi_{00}\right)$ 是末知数外, 其它值都可由 实验测量得到。因此测量调制电荷是一个测量类似 $\mathrm{EV}$ 装犆中电子等离子体的总能量的简单 而有效的方法。

\section{参考文献}

[1] Malmberg, J. H. \& deGrassie, J. S., Phys. Rev. Lett., 35 (1975), 577.

[2] Malmberg, J. H. et al., Physica Seripta, T2 (1982), 288.

[3] deGrassie, J. S, \& Malmberg, J. H., Phys. Fluids, 23 (1980), 63.

[4] O'Neil, T. M., Proc. Inter. Cont. on Plasma Physics, II (1980), 321.

[5] Malmberg, J. H. \& OיNeil, T. M., Phys. Rev. Lett., 39 (1977), 1333.

[6] Malmberg, J. H. \& Driscoll, C. F., ibid., 44 (1980), 654. 